MODELING, IDENTIFICATION AND CONTROL, 1980, vOL. 1, NO. 2, 93-103

doi: $10.4173 /$ mic.1980.23

\title{
On the problem of identification in compartment analysis
}

\author{
ERIK AARNÆS† \\ Keywords: linear compartment system, identification, realization theory, Newton- \\ Raphson method, generalized inverse.
}

\begin{abstract}
The present paper discusses how the impulse response of an unknown linear multi-compartment system can be used to identify the system and points out some of the problems associated with a unique identification. A method for system identification has been implemented in a computer program. Simulated data have been used to study the accuracy of the identification from the impulse response. It is shown that accurate identification of a linear multi-compartment system may require, if no part of the system is known a priori, that the observed impulse response contains the results of several independent experiments in each of which several independent combinations of compartments are observed.
\end{abstract}

\section{Introduction}

The use of compartment analysis in biology and medicine has beer described by, for instance, Atkins (1969) and Jacques (1972). The present paper is restricted to linear compartment systems. Such systems can be described by a set of linear firstorder differential equations, one equation for each compartment. Several processes can be described by linear compartment systems. Examples are diffusion across a membrane and first-order chemical reactions. The fact that many non-linear systems can be approximated by linear systems is also important. This can often be done when studying complex biochemical systems through the use of small amounts of radioactive tracers.

Figure 1 shows a third-order linear compartment system, or a three-compartment system. The state variables $x_{1}, x_{2}$ and $x_{3}$ give the state of the respective compartments. The constants $k_{i, j}(i, j=1,2,3)$ give the relative rates of flow. The constants $k_{i, i}$ $(i=1,2,3)$ give the relative rates of loss.

Using results from linear system theory the paper discusses how to determine the constants of the system from observation of its dynamic behaviour. Numerical examples will be given to show how the results may be utilized. The numerical examples have been generated with the aid of two computer programs. The first program simulates observations with stochastic errors of a linear compartment system. The second program tries to identify the system that lies behind the simulated observations.

\section{The problem of system identification}

Assume that the system to be identified is of order $n$ ( $n$ state variables or compartments). The system may be described by the matrix differential equation

$$
\frac{d x}{d t}=A x
$$

$\dagger$ Institute of Informatics, University of Oslo, P.b. 1080, Blindern. Oslo 3, Norway. Present address: A/S Computas. P.b. 310, 1322 Høvik, Norway. 


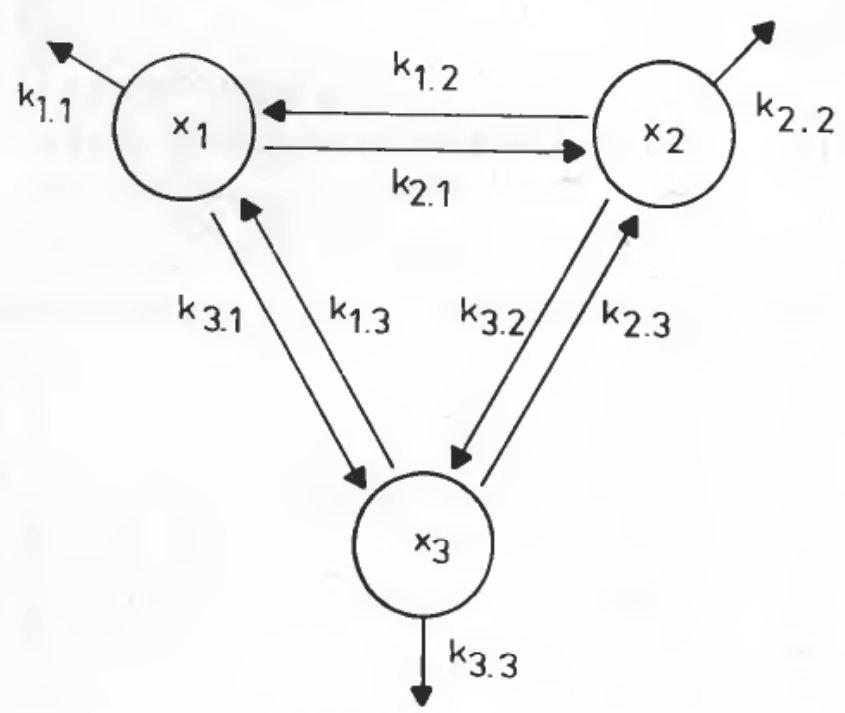

Figure 1. A third-order multi-compartment system.

where $x$ is the $n$-dimensional vector of state variables (the state vector) and $A$ is the $(n \times n)$ system matrix. Assume that $p$ different linear combinations of the state variables are observed. The $p$-dimensional vector $y$ of observable linear combinations is then given by $y=C x$ where $C$ is the $(p \times n)$ observation matrix. Assume that an experiment is performed in which the system is given the initial state $x(0)=b_{1}$, after which the system is left to itself. It then follows from linear system theory (Ogata 1967) that the response $y_{1}(t)=C \exp (A t) b_{1}$ where $\exp (A t)$ is the $(n \times n)$ transition matrix of the system. If $q$ such experiments are performed, this defines the $(n \times q)$ initial value matrix $B=\left[b_{1}, \ldots, b_{q}\right]$ and the $(p \times q)$ impulse response $Y(t)=\left[y_{1}(t), \ldots, y_{q}(t)\right]$. The response of the system to the $q$ experiments can therefore be written

$$
Y(t)=C \exp (A t) B
$$

The problem is to calculate the system matrix $A$ from knowledge of the impulse response $Y(t)$, the observation matrix $C$ and the initial value matrix $B$. Knowledge of $C$ corresponds to knowledge of which combinations of compartments have been observed, while knowledge of $B$ corresponds to knowledge of the initial values of the compartments in each experiment.

This problem has been termed 'the inverse problem' or 'the problem of identification' (Monot and Martin 1974, Jacques 1972, Rubinow and Winzer 1971). In general linear system theory parts of this problem are treated under the name of 'realization theory' (Silverman 1971).

In the following, the system with system matrix $A$, initial value matrix $B$ and observation matrix $C$ will be termed 'the system $(A, B, C)$ ' or ' $(A, B, C)$ '. Equation (2) thus gives the impulse response of the system $(A, B, C)$.

Let $P$ be any invertible $(n \times n)$ matrix, and let

$$
\begin{aligned}
\Lambda & =P^{-1} A P \\
\beta & =P^{-1} B \\
\gamma & =C P \\
z & =P^{-1} x
\end{aligned}
$$


Since $\gamma \exp (\Lambda t) \beta=C \exp (A t) B$ (Silverman 1971, Ogata 1967), the systems $(\Lambda, \beta, \gamma)$ and $(A, B, C)$ have the same impulse response. Even an exact knowledge of the impulse response is therefore not sufficient to determine uniquely the system that produced the impulse response.

Assume that we have succeeded in determining one possible system $(\Lambda, \beta, \gamma)$ with the observed impulse response. If $n$ experiments are performed and $\beta$ and $B$ are known and invertible, $(3 a-c)$ can be used to obtain $A$ and $C$ through the equations $A=B \beta^{-1} \Lambda \beta B^{-1}$ and $C=\gamma \beta B^{-1}$. If instead $\gamma$ and $C$ are known and invertible, $(3 a-c)$ can be used to determine $A$ and $B$ through the equations $A=C^{-1} \gamma \Lambda \gamma^{-1} C$ and $B=C^{-1} \gamma \beta$.

If each compartment is observed on its own, then $C$ is the identity matrix and $A=\gamma \Lambda \gamma^{-1}$. Further, if $(\Lambda, \beta, \gamma)$ is determined in such a way that $\Lambda$ is a diagonal matrix, then $\gamma$ is the matrix whose columns are the eigenvectors of the matrix $A$. In cases where $\beta$ is an $(n \times 1)$ matrix, i.e. only one experiment is performed, this is therefore equivalent to a well-known procedure for the calculation of the system matrix $A$ (Rubinow and Winzer 1971, Norwich 1977).

Complete mathematical symmetry exists between the observation matrix $C$ and the initial value matrix $B$. In spite of this symmetry, $B$ and $C$ are, of course, experimentally obtained in significantly different ways. Situations may be conceived in which parts of the system being studied are unavailable for observation, but available for manipulation. In such cases, lack of knowledge of $C$ or of the behaviour of some compartments may in principle be replaced by independent experiments.

\section{The identification of one possible system}

The problem remains to identify a system $(\Lambda, \beta, \gamma)$ with the desired impulse response. The following solution is an application of results which were presented independently by Kalman (1963) and Gilbert (1963). The solution is restricted to linear systems whose dynamic behaviour can be described by linear combinations of exponential functions, but was selected because it is readily adapted to a least-squares fitting of a theoretical impulse response to an observed response with errors of measurement.

Assume that the impulse response of an unknown system has been observed at the times $t_{1}, t_{2}, \ldots, t_{N}$. We wish to determine an $n$th order system (preselected value of $n$ ) with an impulse response close to the observed impulse response. Since the impulse response $Y(t)$ of the unknown system can be described by linear combinations of exponential functions, we shall fit

$$
Y(t)=\sum_{i=1}^{n} H_{i} \exp \left(\lambda_{i} t\right)
$$

to the observations. Since $Y(t)$ is a $(p \times q)$ matrix of functions, $H_{i}$ are $(p \times q)$ matrices of coefficients of the scalar exponential functions $\exp \left(\lambda_{i} t\right)(i=1,2, \ldots, n)$. Therefore (4) specifies $p q$ linear combinations of exponential functions. Let $r_{i}$ be the rank (the number of independent rows or columns) of $H_{i}$. Gilbert (1963) and Kalman (1963) showed that the minimal order $m$ of a system with the impulse response (4) is given by

$$
m=\sum_{i=1}^{n} r_{i}
$$

and that $\lambda_{i}$ is an eigenvalue of multiplicity $r_{i}$. Since we want $m=n$, it follows from (5) 
that we must require $r_{i}=1$ for all values of $i$. If some $r_{i}>1$, the order (number of compartments) of the resulting system will be greater than $n$.

If the impulse response is describing only one experiment $(q=1)$ or contains only one linear combination of compartments $(p=1)$, then each $H_{i}$ is a $(p \times 1)$ or $(1 \times q)$ matrix, which must have rank 1 , and the system resulting from (4) will be of order $n$. If, however, $p>1$ and $q>1$, the ranks $r_{i}$ will in general be greater than 1 , and the resulting systems will be of too great order.

Let

$$
H_{i}=\gamma_{i} \beta_{i}
$$

with $\gamma_{i}$ a $(p \times 1)$ matrix and $\beta_{i}$ a $(1 \times q)$ matrix. In this case $H_{i}$ is of rank less than or equal to 1 (if $H_{i}=0$ then $r_{i}=0$ ). If therefore $Y(t)$ is expressed as

$$
Y(t)=\sum_{i=1}^{n} \gamma_{i} \beta_{i} \exp \left(\lambda_{i} t\right)
$$

the requirement that $Y(t)$ should be the impulse response of an $n$th order system is met. It follows that the system $(\Lambda, \beta, \gamma)$ with $\Lambda$ a diagonal matrix $\Lambda=\operatorname{diag}\left(\lambda_{1}, \ldots, \lambda_{n}\right)$, $\beta$ a $(n \times q)$ matrix with rows $\beta_{1}, \ldots, \beta_{n}$ and $\gamma$ a $(p \times n)$ matrix with columns $\gamma_{1}, \ldots, \gamma_{n}$ has the desired impulse response (Gilbert 1963, Kalman 1963).

\section{Fitting the theoretical impulse response to observations}

An alternative way of writing (7) is

$$
y_{l, j}(t)=\sum_{k=1}^{n} \gamma_{l, k} \beta_{k, j} \exp \left(\lambda_{k} t\right)
$$

where $y_{i, j}(t)$ is the $i$, jth element of $Y(t), \gamma_{i, k}$ is the $i, k$ th element of $\gamma$ and $\beta_{k, j}$ is the $k, j$ th element of $\beta$.

We want to fit (8) to the $K=N p q$ observations $\hat{y}_{i, j}\left(t_{l}\right)(i=1, \ldots, p ; j=1, \ldots, q$; $l=1, \ldots, N)$. A unique correspondence between $s=1, \ldots, K$ and the triples $i, j, l$ can be specified giving $\xi_{s}=y_{i, j}\left(t_{l}\right)$ and $\hat{\xi}_{s}=\hat{y}_{i, j}\left(t_{l}\right)$. Let the number of parameters defining (8) be $M$, where $M=n+n p+n q$. We can then specify a unique correspondence between $\omega_{v}(v=1, \ldots, M)$ and $\lambda_{k}, \beta_{k, j}$ and $\gamma_{i, k}(i, j$ and $k$ as above). Equation (8) may now be rearranged into the set

$$
\left.\begin{array}{c}
\xi_{1}=\Phi_{1}\left(\omega_{1}, \ldots, \omega_{M}\right) \\
\vdots \\
\xi_{K}=\Phi_{K}\left(\omega_{1}, \ldots, \omega_{M}\right)
\end{array}\right\}
$$

Equation (9) can be linearized around some set of values of the $\omega_{v}$, say $\omega_{v}^{(j)}$. This gives for $s=1, \ldots, K$

$$
\xi_{s} \approx \Phi_{s}\left(\omega^{(j)}\right)+\left[\frac{\partial \Phi_{s}^{(j)}}{\partial \omega_{1}}, \ldots, \frac{\partial \Phi_{s}^{(j)}}{\partial \omega_{M}}\right]\left[\begin{array}{c}
\Delta \omega_{1}^{(j)} \\
\vdots \\
\Delta \omega_{M}^{(j)}
\end{array}\right]
$$

where

$$
\omega^{(j)}=\left[\omega_{1}^{(j)}, \ldots, \omega_{M}^{(j)}\right]^{\mathrm{T}} \text { and } \Delta \omega^{(j)}=\omega-\omega^{(j)}
$$


The problem is now to minimize

$$
\sum_{s=1}^{K} \sigma_{s}\left\{\left[\frac{\partial \Phi_{s}^{(j)}}{\partial \omega_{1}}, \ldots, \frac{\partial \Phi_{s}^{(j)}}{\partial \omega_{M}}\right]\left[\begin{array}{c}
\Delta \omega_{1}^{(j)} \\
\vdots \\
\Delta \omega_{M}^{(j)}
\end{array}\right]+\Phi_{s}\left(\omega^{(j)}\right)-\hat{\xi}_{s}\right\}^{2}
$$

with respect to $\omega . \sigma_{s}$ can for instance be $1 / \operatorname{var}\left(\hat{\xi}_{s}\right)$ where $\operatorname{var}\left(\hat{\xi}_{s}\right)$ denotes the variance of the observation $\hat{\xi}_{s}$.

Let $\theta_{j}$ be the $(K \times M)$ matrix of partial derivatives, i.e. the Jacobian matrix with the $s$, lth element $\partial \Phi_{s}^{(j)} / \partial \omega_{l}$. The minimization of (11) can now be done through the iterative procedure

$$
\begin{aligned}
\theta_{j}^{\mathrm{T}} \Sigma \theta_{j} \Delta \omega^{(j)} & =\theta_{j}^{\mathrm{T}} \Sigma \Delta \xi^{(j)} \\
\omega^{(j+1)} & =\omega^{(j)}+\Delta \omega^{(j)}
\end{aligned}
$$

where

$$
\Sigma=\operatorname{diag}\left(\sigma_{1}, \ldots, \sigma_{K}\right) \text { and } \Delta \xi^{(j)}=\left[\left(\Phi_{1}\left(\omega^{(j)}\right)-\hat{\xi}_{1}\right), \ldots,\left(\Phi_{K}\left(\omega^{(j)}\right)-\hat{\xi}_{K}\right)\right]^{\mathrm{T}}
$$

The above iterative method is essentially the minimization of

$$
J(\omega)=\sum_{s=1}^{K} \sigma_{s}\left(\Phi_{s}(\omega)-\hat{\xi}_{s}\right)^{2}
$$

by solving the set

$$
\frac{\partial J}{\partial \omega_{l}}(\omega)=0 \quad(l=1, \ldots, M)
$$

Since (14) is non-linear, it must be solved iteratively. The resulting linearization gives a set of equations including the second derivatives $\partial^{2} \Phi_{s} / \partial \omega_{i} \partial \omega_{j}(i, j=1, \ldots, M)$, which are tedious to determine. It is easy to show that if the terms including the second derivatives are neglected, the resulting set of equations is identical to $(12 a)$.

The use of $(12 a)$ requires that the matrix $\theta_{j}{ }^{\mathrm{T}} \Sigma \theta_{j}$ is invertible at each step $j$ of iteration. Unfortunately this is not true in the present case because the factorization in (6) is not unique. If (6) is true, then so is $H_{i}=\left(\gamma_{i} / \rho_{i}\right)\left(\rho_{i} \gamma_{i}\right)$ where $\rho_{i}$ is any number different from zero. Because of this $(12 a)$ does not have a unique solution. This also applies to (14). Since there are $n$ non-unique factors $\rho_{i}$, the rank of the $(M \times M)$ matrix $\theta_{j}^{\mathrm{T}} \Sigma \theta_{j}$ will be less than or equal to $(M-n)$. However, $(12 a)$ can be solved by using the generalized inverse (Penrose 1955, 1956, Jackson 1972). The generalized inverse is computed using an IMSL-library routine (IMSL 1977) for the singular value decomposition of a matrix (Golub and Reinsch 1970). A rigorous treatment of the convergence of the Newton-Raphson method when the Jacobian matrix is noninvertible and the generalized inverse is used, has been given by Ben-Israel (1966).

It should be noted that $(12 a)$ can be replaced by the overconstrained set

$$
\Sigma^{1 / 2} \theta_{j} \Delta \omega^{(j)}=\Sigma^{1 / 2} \Delta \xi^{(j)}
$$

At each iteration (15) could be solved directly by using the generalized inverse. This reduces rounding errors compared to $(12 a)$ Golub and Reinsch 1970). Equation $(12 a)$ was used in the present case, however, because the $(K \times M)$ matrix $\Sigma^{1 / 2} \theta_{j}$ would have required a very large space in the computer. Since $M$ is much smaller than 
$K$ the $(M \times M)$ matrix $\theta_{j}{ }^{\mathrm{T}} \Sigma \theta_{j}$ requires much less space. If the latter consideration does not arise, however, (15) should be used instead of (12a), in particular when (12a) (and (15)) is ill-conditioned.

\section{Results}

Numerical examples were constructed by simulating the impulse response of a given system $(A, B, C)$. Stochastic errors were introduced by multiplication with normally distributed pseudo-random numbers with mean 1 and selected standard deviation. Exponential functions were then fitted to the simulated impulse response as indicated in $\S 4$. The system matrix was, when possible, calculated according to $(3 a-c)$.

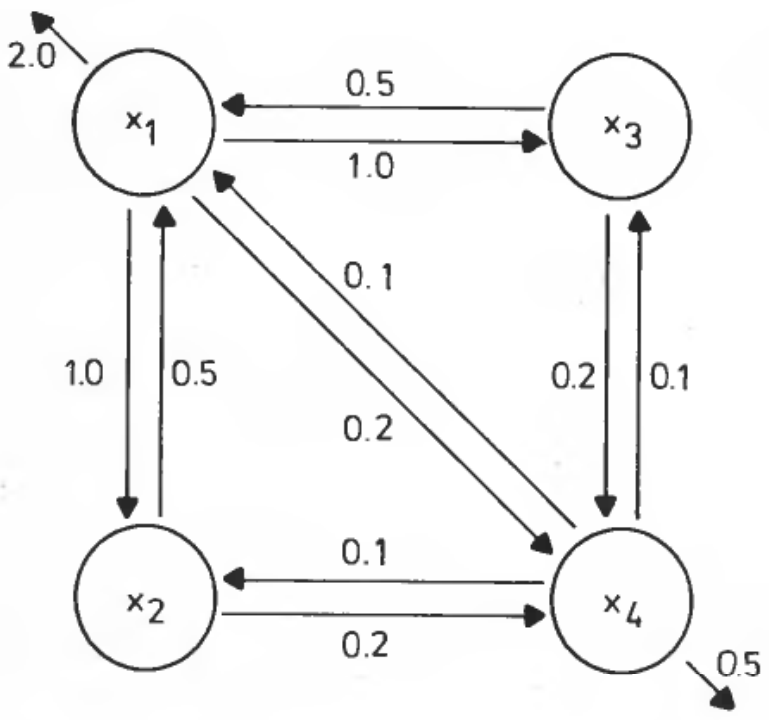

Figure 2. The test system used to investigate possible practical results of the mathematical analysis. There are losses from compartments 1 and 4, but not from 2 and 3 . There are direct connections between all compartments except between 2 and 3 .

The test system is presented in Fig. 2. This system corresponds to the system matrix

$$
A=\left[\begin{array}{rrrr}
-4.2 & 0.5 & 0.5 & 0.1 \\
1.0 & -0.7 & 0.0 & 0.1 \\
1.0 & 0.0 & -0.7 & 0.1 \\
0.2 & 0.2 & 0.2 & -0.8
\end{array}\right]
$$

with the four eigenvalues $-0 \cdot 322,-0 \cdot 700,-0 \cdot 911,-4 \cdot 467$.

The first test was made on an impulse response with an accuracy of three digits. An observation matrix corresponding to independent observation of each compartment was used. The initial value matrix that was used corresponds to four experiments, 
in each of which a different compartment is given the initial value 1 , the others the initial value 0 . This is achieved if the observation matrix $C$ and the initial value matrix $B$ both equal the identity matrix. The resulting impulse response is indicated in Fig. 3.
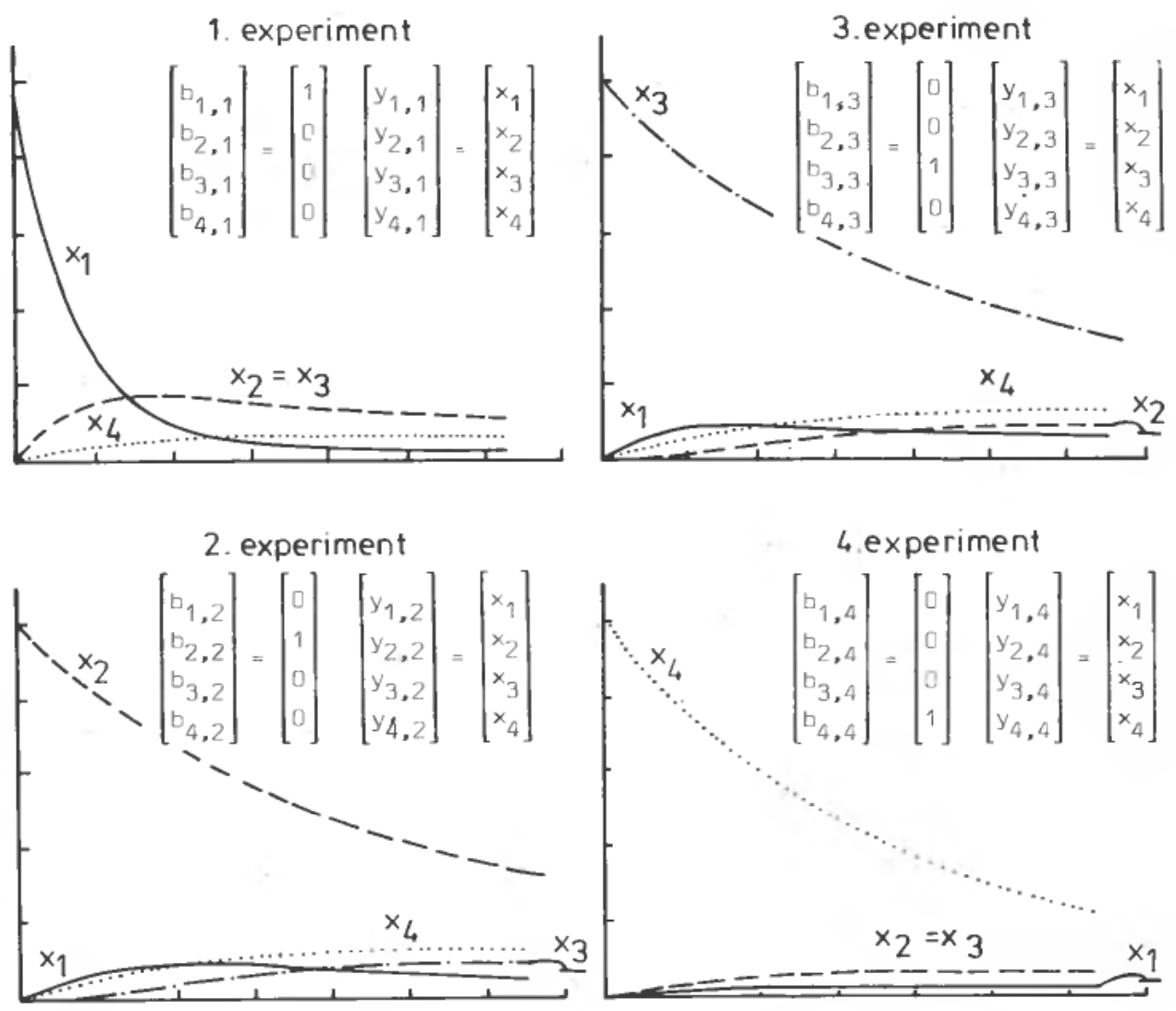

Figure 3. The impulse response of the four-compartment system of Fig. 2. The curves show the simulated responses of each compartment after four independent experiments. In each experiment a different compartment is given the initial value 1 , the remaining compartments the initial value 0 .

The computed impulse response matrix $A_{\mathrm{c}}$ is

$$
A_{\mathrm{c}}=\left[\begin{array}{rrrr}
-4.19966 & 0.49976 & 0.49976 & 0.10002 \\
0.99984 & -0.69983 & 0.00007 & 0.10001 \\
0.99984 & 0.00007 & -0.69983 & 0.10001 \\
0.20002 & 0.20000 & 0.20000 & -0.80016
\end{array}\right]
$$

The 16 curves were each sampled at 51 points, a total of $51 \times 16=816$ points. When compared, (16) and (17) shows a near identity. The method of the present paper therefore seems to give good results, at least when the system is readily accessible for accurate measurements. 


\begin{tabular}{|c|c|c|c|c|c|c|c|}
\hline Example & Observat & tion 1 & matrix & \multicolumn{4}{|c|}{ Computed system matrix } \\
\hline \multirow{4}{*}{1} & \multirow{5}{*}{$\begin{array}{l}1 \\
0 \\
0\end{array}$} & 10 & 07 & {$[-4 \cdot 013$} & 0.461 & 0.451 & 0.0957 \\
\hline & & 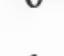 & 1 & 0.941 & -0.684 & 0.018 & $0 \cdot 101$ \\
\hline & & 1 & & 0.960 & 0.009 & -0.687 & $0 \cdot 102$ \\
\hline & & 0 & 1] & 0.199 & $0 \cdot 194$ & $0 \cdot 193$ & -0.803 \\
\hline \multirow{4}{*}{2} & & & & {$[-4 \cdot 100$} & $0 \cdot 487$ & 0.567 & $0 \cdot 111]$ \\
\hline & {$\left[\begin{array}{ll}1 & 1\end{array}\right.$} & 0 & 07 & 0.961 & -0.697 & -0.044 & 0.097 \\
\hline & {$\left[\begin{array}{ll}0 & 0\end{array}\right.$} & 1 & 0 & 0.961 & 0.000 & -0.753 & 0.098 \\
\hline & & & & L.294 & $0 \cdot 215$ & $0 \cdot 370$ & $-0.804]$ \\
\hline \multirow{4}{*}{3} & \multirow{4}{*}{1} & \multirow{4}{*}{\multicolumn{2}{|c|}{$\left.\begin{array}{ll}0 & 0\end{array}\right]$}} & {$[-4 \cdot 150$} & $0 \cdot 486$ & $0 \cdot 547$ & 0.0957 \\
\hline & & & & 0.995 & -0.699 & -0.035 & $0 \cdot 104$ \\
\hline & & & & $0 \cdot 309$ & $-0 \cdot 121$ & $-0 \cdot 380$ & $0 \cdot 195$ \\
\hline & & & & $1 \cdot 274$ & $0 \cdot 414$ & -0.229 & -0.960 \\
\hline \multirow{4}{*}{$3 b$} & \multirow{4}{*}{1} & \multirow{4}{*}{\multicolumn{2}{|c|}{$\left.\begin{array}{ll}0 & 0\end{array}\right]$}} & {$[-4 \cdot 103$} & 0.441 & $0 \cdot 552$ & $0 \cdot 1017$ \\
\hline & & & & $0 \cdot 978$ & -0.683 & -0.034 & $0 \cdot 105$ \\
\hline & & & & $0 \cdot 249$ & $0 \cdot 100$ & -0.556 & 0.043 \\
\hline & & & & $1 \cdot 363$ & $0 \cdot 071$ & 0.035 & -0.732 \\
\hline \multirow{4}{*}{4} & \multirow{4}{*}{1} & \multirow{4}{*}{$\begin{array}{ll}0 & 0\end{array}$} & \multirow{4}{*}{ 0] } & {$[-4 \cdot 187$} & $0 \cdot 494$ & 0.510 & 0.0987 \\
\hline & & & & $3 \cdot 612$ & -0.878 & -0.635 & 0.084 \\
\hline & & & & -1.656 & $0 \cdot 165$ & -0.202 & $0 \cdot 145$ \\
\hline & & & & 0.331 & $0 \cdot 238$ & $0 \cdot 520$ & $-0.864]$ \\
\hline \multirow{4}{*}{5} & \multirow{4}{*}{1} & \multirow{4}{*}{11} & \multirow{4}{*}{ 1] } & {$[-3 \cdot 406$} & 0.374 & $0 \cdot 294$ & -0.4737 \\
\hline & & & & $1 \cdot 503$ & -0.782 & -0.344 & 0.026 \\
\hline & & & & -0.031 & $0 \cdot 181$ & $-0 \cdot 110$ & 0.442 \\
\hline & & & & 0.411 & $0 \cdot 145$ & -0.011 & -0.717 \\
\hline \multirow{4}{*}{6} & \multirow{4}{*}{$\begin{array}{ll}1 & 1 \\
0 & 0 \\
0 & 0\end{array}$} & \multirow{4}{*}{0} & 0 & {$[-4 \cdot 166$} & 0.493 & $0 \cdot 488$ & 0.0997 \\
\hline & & & 0 & 0.989 & -0.697 & 0.004 & $0 \cdot 100$ \\
\hline & & & & 0.993 & 0.002 & -0.697 & $0 \cdot 100$ \\
\hline & & & 1] & $0 \cdot 200$ & $0 \cdot 199$ & $0 \cdot 199$ & -0.800 \\
\hline
\end{tabular}


The program was then tested on simulated data with a relative standard deviation of $5 \%$. The results are given in the Table. The same system matrix and the same initial value matrix, but different observation matrices were used in the Examples of the Table (except for Examples 3 and $3 \mathrm{~b}$ ). The total number of observations (the number of experiments $\times$ the number of observed curves per experiment $\times$ the number of samples per observed curve) was approximately constant (from 484 in Example 5 to 496 in Example 1). The empirical standard deviation was computed from the difference between the computed and the observed impulse response after each least squares fit of the model to the data. In each case the empirical standard deviation was not significantly different from the standard deviation of the simulated errors.

In Example 1, the computed system matrix is in good agreement with the answer. The three rows of the observation matrix indicate observation of three different combinations of compartments; the sum of compartments 1 and 2, compartment 3 alone and compartment 4 alone. In Examples 2 and 3 compartment 4 and compartments 3 and 4 respectively are not included in the impulse response. Row 4 in Example 2, and rows 3 and 4 in Example 3 show little or no agreement with the true answer. In spite of the fact that the total number of observations is approximately the same in the three Examples, the agreement between the computed system matrix and the test system is therefore reduced when the test system is less available for direct observation.

Example $3 b$ is a repetition of Example 3, but with different initial values for the iterations of $(12 a, b)$. Rows 1 and 2 are in good agreement, but rows 3 and 4 show no agreement in the two examples, demonstrating that the latter rows cannot be determined accurately from the simulated observations of Examples 3 and $3 b$. The goodness of the least-squares fit is almost independent of rows 3 and 4 in the system matrix.

The computed system matrix of Example 5 shows no agreement with the correct answer. In this example the observation matrix corresponds to the observation of the sum of all compartments, for instance total radioactivity in a tracer experiment.

Example 6 is similar to Example 1, except for the errors in the simulated observations. In Example 6 the relative standard deviation is $1 \%$, as opposed to $5 \%$ in Example 1. The difference between the computed system matrix and the test system is correspondingly reduced.

\section{Discussion}

It is important to distinguish between the following parts of compartment analysis:

(i) to describe a system as a multi-compartment system and simulate the system (solve the differential equations) in order to investigate the behaviour of the system; and

(ii) from observation of the behaviour of an unknown system, to identify the system (find the differential equations describing the system).

These parts are significantly different both in their nature and in their degree of difficulty. When the system, or a set of assumptions describing the system, is known, (i) is in principle straightforward. Part (ii) is the non-trivial part of compartment analysis. In general a system which is consistent with the observations can be found. The difficult part of the problem is the uniqueness of the solution. This is of general 
importance in the use of mathematical models to describe natural systems. It is important to realize that several models may fit a given set of data equally well. For instance, a different linear model with an identical impulse response can always be found by increasing the order (the number of compartments) of the model. Only a minimal system (a system with the minimal number of compartments, or equivalently, an observable and controllable system) can, at best, be uniquely determined.

Most methods for system identification only utilize a single experiment (the initial value matrix is an $(n \times 1)$ matrix $)$. In such cases, unique identification of the system requires, if no part of the system matrix is known a priori, that $n$ independent combinations of compartments are observed (the observation matrix must be invertible). The present work indicates, however, that a significantly better identification can be achieved if the model is simultaneously fitted to different experiments. The numerical examples suggest that one may expect to identify the system with the same accuracy as the data if the entire (or 'almost' entire) impulse response has been observed. For an $n$-compartment system, the entire impulse response corresponds to $n$ independent experiments, in each of which $n$ independent combinations of compartments are observed. As shown in $\S 3$, this puts restrictions on how theoretical curves should be fitted to the observations.

In all the numerical examples in $\S 5$ the initial value matrix $B$ is the $(4 \times 4)$-identity matrix. In terms of linear system theory all the test systems are therefore controllable. In Examples 4 and 5 one of the eigenvalues $(-0.7)$ is not present in the simulated impulse response. In terms of linear system theory these test systems are therefore not observable. Example 4 indicates, however, that parts of an unobservable system may at least occasionally be uniquely identified. Example 4 consists of four experiments in which each compartment in turn is given the initial value 1 . Assuming that the initial slope of the curve describing compartment 1 is known in each experiment, we obtain

$$
\begin{aligned}
& \dot{x}_{1,1}(0)=-\left(k_{1,1}+k_{2,1}+k_{3,1}+k_{4,1}\right) \\
& \dot{x}_{1,2}(0)=k_{1,2} \\
& \dot{x}_{1,3}(0)=k_{1,3} \\
& \dot{x}_{1,4}(0)=k_{1,4}
\end{aligned}
$$

where $\dot{x}_{1, i}(0)(i=1,2,3,4)$ denotes the four initial slopes. If these initial slopes can be determined with reasonable accuracy, the first line of the system matrix can be found. This example may seem obvious, but it shows that observability need not be a requirement for partial identification.

\section{REFERENCES}

AtKIns, G. L. (1969). Multicompartment Models for Biological Systems (Methuen).

BEN-ISRAEL, A. (1966). A Newton-Raphson method for the solution of systems of equations. J. math. Analysis Applic., 15, 243-252.

GILBERT, E. G. (1963). Controllability and observability in multivariable control systems. S.I.A.M. J. Control, 1, 128-151.

Golub, G. H., and ReINSCH, C. (1970). Singular value decomposition and least squares solutions. Num. Math., 14, 403-420.

IMSL-REFERENCE MANUAL (1977). IMSL-library 2, edition 6. IMSL, GNB Building, 7500 Bellaire Boulevard, Houston, Texas 77036, U.S.A.

JACKSON, D. D. (1972). Interpretation of inaccurate, insufficient and inconsistent data. Geophys. J. R. astr. Soc., 28, 97-109. 
JACQUES, J. A. (1972). Compartmental Analysis in Biology and Medicine (Elsevier).

KalmaN, R. E. (1963). Mathematical description of linear dynamical systems. S.I.A.M. J. Control, 1, 152-192.

Monot, C., and MarTin, J. (1974). Reflections on some algorithms for the solution of the inverse problem (identification or adjustment) for linear compartment models. Mathematical Models in Biology and Medicine, edited by N. T. J. Bailey, Bl. Sendov and R. Tsanev (North-Holland), pp. 49-70.

Norwich, K. H. (1977). Molecular dynamics in biosystems. The Kinetics of Tracers in Intact Organisms (Pergamon Press), p. 119.

Ogata, K. (1967). State Space Analysis of Control Systems (Prentice-Hall).

Penrose, R. (1955). A generalized inverse for matrices. Proc. Camb. phil. Soc., 51, 406-413; (1956). On best approximate solution of linear matrix equations. Proc. Camb. phil. Soc. 52, $17-19$.

Rubinow, S. I., and Winzer, A. (1971). Compartment analysis: an inverse problem. Math. Biosci., 11, 203-247.

Silverman, L. M. (1971). Realization of linear dynamical systems. I.E.E.E. Trans. autom. Control, 16, 554-567. 